\title{
SPECTROPOLARIMETRY OF THE TYPE Ia SN 2007sr TWO MONTHS AFTER MAXIMUM LIGHT*
}

\author{
P. Zelaya ${ }^{1}$, J. R. Quinn ${ }^{1}$, D. BaAde ${ }^{2}$, A. Clocchiatti ${ }^{1}$, P. Höflich $^{3}$, J. Maund $^{4}$, F. Patat $^{2}$, L. WAng $^{5}$, And J. C. Wheeler $^{6}$ \\ ${ }^{1}$ Department of Astronomy, Pontificia Universidad Católica de Chile, Casilla 306, Santiago 22, Chile; pazelaya@ astro.puc.cl \\ ${ }^{2}$ ESO-European Organization for Astronomical Research in the Southern Hemisphere, Karl-Schwarzschild-Str.2, D-85748 Garching bei München, Germany \\ ${ }^{3}$ Department of Physics, Florida State University, Tallahassee, FL 32306-4350, USA \\ ${ }^{4}$ Queen's University Belfast, University Road, Belfast BT7 1NN, UK \\ ${ }^{5}$ Department of Physics, Texas A\&M University, College Station, TX 77843-4242, USA \\ ${ }^{6}$ Department of Astronomy and McDonald Observatory, The University of Texas at Austin, 1 University Station, C1400, Austin, TX 78712, USA \\ Received 2012 April 27; accepted 2012 November 4; published 2012 December 13
}

\begin{abstract}
We present late-time spectropolarimetric observations of SN 2007sr, obtained with the Very Large Telescope at the ESO Paranal Observatory when the object was 63 days after maximum light. The late-time spectrum displays strong line polarization in the Ca II absorption features. SN 2007sr adds to the case of some normal Type Ia supernovae that show high line polarization or repolarization at late times, a fact that might be connected with the presence of high-velocity features at early times.
\end{abstract}

Key words: supernovae: individual (SN 2007sr) - techniques: polarimetric

Online-only material: color figures

\section{INTRODUCTION}

Type Ia supernovae ( $\mathrm{SNe}$ ) are believed to form in binary systems. Traditional scenarios involve a carbon-oxygen white dwarf accreting material from a companion, which could be a main sequence, a red giant, a helium, or another white dwarf star. As the degenerate star accretes material, it approaches the Chandrasekhar mass limit. Once it arrives at that point, it becomes pressure unstable and a thermonuclear runaway starts at one or more ignition points. A thermonuclear burning front propagates synthesizing new elements, at first subsonically in a deflagration regime, and then it probably experiences a transition to a supersonic regime, e.g., a detonation (Khokhlov 1991; Hillebrandt et al. 2000). The deflagration phase leaves a residual of intermediate-mass elements. In the detonation, the thermonuclear burning continues until nuclear statistical equilibrium is reached. The thermonuclear burning of a white dwarf is a complex process and the details are still subject to debate.

In the last few decades, Type Ia $\mathrm{SNe}$ have successfully proved to be reliable distance indicators when standardized by their luminosity decline rate (Phillips 1993; Hamuy et al. 1996; Prieto et al. 2005). They represent a rather homogeneous class, in particular the subtype defined by Branch et al. (1993) as "spectroscopically normal" Type Ia SNe. Some diversity still remains in their peak luminosity, color, and light-curve shape, and this is attributed to different intrinsic SN properties such as progenitors, host galaxy, environment, and explosion mechanism (Ivanov et al. 2000; Hamuy et al. 2000; Höflich \& Khokhlov 1996).

The natural assumption of spherical symmetry, which is implicit in the use of SNe as distance estimators, can be tested by using different tools such as (1) powerful hydrodynamical simulations, (2) nebular phase spectroscopy, when the ejecta becomes transparent, the continuum intensity decreases and emission lines from the deep interior of the exploded star emerge, and (3) high-quality spectropolarimetric observations collected

\footnotetext{
* Based on observations obtained with the VLT at the European Southern Observatory (ESO), Chile (ESO program 080.D-0107A).
}

throughout the last decade. Starting from a spherically symmetric white dwarf, hydrodynamical models produce asymmetries by off-center ignition and highly turbulent nuclear burning during the deflagration phase (Kasen et al. 2008, 2009). Coupled with viewing angle effects these contribute to observational diversity. Nebular phase spectroscopy, starting at about 100 days after maximum light, reveals the inner core of the $\mathrm{SN}$ with signatures of asymmetries formed in these stages (Maund et al. 2010a; Maeda et al. 2010b). Hubble Space Telescope imaging of the SN remnant SN1885, S-Andromeda, revealed asymmetries in $\mathrm{Ca}$ and $\mathrm{Fe}$ that can be understood in the framework of off-center delayed detonations (Fesen et al. 2007). Spectropolarimetric observations show large diversity, except perhaps in the continuum polarization, where low values are common. According to theoretical interpretation (Höflich 1991; Howell et al. 2001), this indicates that the photosphere is not completely spherical, and small global asymmetries, less than $15 \%$, are inferred. A contrasting picture is revealed by the polarization in the blueshifted absorption troughs of the P Cygni profiles where a large diversity is observed. This is usually interpreted as the result of smallscale departures of symmetry such as chemical or excitation inhomogeneities. Spectropolarimetric data show line polarization for different elements, such as Si II, Ca II, and $\mathrm{S}_{\text {II, }}$ with different values at different epochs of the $\mathrm{SN}$ evolution. The general consensus emerging from the data is that Type Ia SNe are more asymmetric in their outer layers and more spherically symmetric in the interior. This implies that line polarization and continuum polarization are higher at early times and decrease toward later epochs when the photosphere recedes into the inner layers (Filippenko \& Leonard 2004; Leonard et al. 2005; Wang \& Wheeler 2008; Wang et al. 2003). However, SN 2006X, one of the few Type Ia SNe that have been observed in spectropolarimetry at times later than 30 days after maximum, does show strong late-time line polarization in the Ca II IR triplet (Patat et al. 2009).

In this paper, we report spectropolarimetric observations of SN 2007sr when the SN was more than two months after maximum and showed highly polarized Ca II lines. In Section 2 we present the data, in Section 3 we describe the results, and in Section 4 we discuss them and present our conclusions. 


\section{DATA}

\subsection{Supernova 2007sr}

SN 2007sr was discovered in the Antennae galaxies (NGC 4038/39) on December 18.53 UT by the Catalina Sky Survey (Drake et al. 2007). It was classified as a Type Ia about three to four days after $B$ maximum light (Pojmanski et al. 2008; Umbriaco et al. 2007). It spectroscopically matched normal Type Ia $\mathrm{SNe}$ with an Si II velocity gradient $\dot{v}_{\mathrm{Si} \text { II }}=$ $80 \pm 15 \mathrm{~km} \mathrm{~s}^{-1}$ day $^{-1}$ (Maeda et al. 2010a), which following Benetti et al. (2005) places it among the high-velocity gradient (HVG) events. Schweizer et al. (2008) calibrate the intrinsic brightness of SN 2007sr using the analysis package SNooPy (Burns et al. 2011) and, following Folatelli et al. (2010), assume a low value for the ratio of total-to-selective extinction $R_{V}^{\text {host }}=1.6 \pm 0.1$. They find a decline-rate parameter $\Delta m_{15}=$ $0.97 \mathrm{mag} \pm 0.02 \mathrm{mag}$ and a color excess in the host galaxy $E(B-V)_{\text {host }}=0.13 \mathrm{mag} \pm 0.01 \mathrm{mag}$. The color excess caused by our own galaxy is $E(B-V)_{M W}=0.046 \pm 0.005$, according to Schlegel et al. (1998).

The distance to SN 2007sr found by Schweizer et al. (2008) is $D=22.3 \pm 2.8 \mathrm{Mpc}$. This makes the host consistent with the large-scale flow model of Tonry et al. (2001), in agreement with four other nearby galaxies that, according to their recession velocities, belong to the same group (Madore \& Steer 2007). Some discrepancy was found with the result of Saviane et al. (2008), who estimated a much smaller distance using the tip of the red giant branch, $D=13.3 \pm 1.0 \mathrm{Mpc}$. The difference in the distance estimates has no effects on the results presented here.

\subsection{Spectropolarimetry of SN $2007 \mathrm{sr}$}

Spectropolarimetric observations of SN 2007sr were obtained with the ESO Very Large Telescope (VLT) UT1, on 2008 February 15 using the FORS 1 instrument in polarimetry mode. ${ }^{7}$ According to the light curve measured by the Carnegie Supernova Project (Table 1 in Schweizer et al. 2008) our observations took place when the SN was 63 days after $B$ maximum light, which corresponds to the spectroscopic Fe II phase (Branch et al. 2005, 2008). At this epoch the spectrum of Type Ia is mainly shaped by the Fe II, Ca II, and Na II lines in addition to forbidden lines.

Four exposures of $\sim 1200 \mathrm{~s}$ were taken with the retarder plate at position angles of $0^{\circ}, 22.5^{\circ}, 45^{\circ}$, and $67.5^{\circ}$. Grism GRIS_300V was used with no order separation filter. This provides a dispersion of $3.28 \AA$ pixel $^{-1}$ and the resulting wavelength range $350-900 \mathrm{~nm}$. The spectral resolution with a $1^{\prime \prime}$ slit is $12.5 \AA$, which we measured using the O I 5577 sky emission line. Additional observations of a flux standard, GD 108 , were performed at a position angle of $0^{\circ}$ to calibrate the flux level.

We followed a standard data reduction procedure. Bias, prescan, and overscan corrections, flat fielding, spectrum extraction, and wavelength calibration were performed using IRAF. ${ }^{8}$ The sensitivity curve of the spectrograph was computed from the flux standard observed at one position angle.

Polarized spectra and flux calibration were obtained with our own IDL routines. Stokes parameters were calculated as described in Patat \& Romaniello (2006). As a starting point,

\footnotetext{
7 For details of the instrument check the FORS Manual at http://www.eso.org/sci/facilities/paranal/instruments/fors/doc/

8 http://iraf.noao.edu-IRAF is distributed by the National Optical Astronomy Observatory, which is operated by the Association of Universities for Research in Astronomy, Inc., under cooperative agreement with the National Science Foundation.
}

a bin size has to be selected. This is a critical step. When polarization is present it should be detected using any bin size, but the estimated polarization values, in particular the peak values, are sensitive to the bin size. We have chosen a bin size of $15 \AA$, slightly larger than the resolution. The spectrum at a position angle of zero was binned to $15 \AA$ and the spectra at the other position angles were resampled to these same bins. The resampling was done using a five-point closed Newtonian-Cotes algorithm.

Observed Stokes parameters are calculated using the formulae given in the VLT FORS User Manual, including the correction for the position angle zero point. The sensitivity function is applied to each beam, together with atmospheric extinction corrections for Paranal as suggested by Patat et al. (2011). The uncertainties of the Stokes parameters $Q$ and $U$ were calculated assuming a Gaussian distribution for the counts at each wavelength.

\section{RESULTS}

\subsection{Intensity Spectrum}

Figure 1 shows the observed intensity spectrum in counts and the signal-to-noise ratio $(\mathrm{S} / \mathrm{N})$. The latter $(\mathrm{S} / \mathrm{N} \sim 100-300)$ is rather low for the standards of spectropolarimetry (generally $\mathrm{S} / \mathrm{N}>500)$, as a result of the $\mathrm{SN}$ being dimmer than estimated when observations were done $\left(M_{B} \sim 16.2\right)$. We note that in the red portion of the spectrum, approximately from $700-950 \mathrm{~nm}$, some of the sky emission lines approach or exceed the SN flux. Background contamination can be an issue there. The blueshifted absorption minimum of the Ca II IR triplet P Cygni profile, in particular, reaches just a few thousand counts and is contaminated by sky emission light in a large fraction of the redder part. Yet some $100 \AA$ of the bluest edge of the absorption feature contain signal above the sky with $\mathrm{S} / \mathrm{N} \sim 60$. This range between 8150 and $8250 \AA$ will be considered to be a reliable zone to measure line polarization.

In Figure 2, we display the intensity spectrum of SN 2007sr and compare it with those of other Type Ia SNe at a similar phase. Following line identifications from the literature (Liu et al. 1997; Hatano et al. 1999; Stanishev et al. 2007) we identify lines of $\mathrm{Si}$ II, Ca II, Na I, Fe II, Fe III, Co II, Co III, and O I at velocities around $13000-10000 \mathrm{~km} \mathrm{~s}^{-1}$, as indicated in the figure.

We detect two systems of weak and narrow Na I D lines in absorption, unresolved by our spectrograph (see Figure 3). One of them, at an observed wavelength of $589.19 \mathrm{~nm}$, is consistent with interstellar matter in the Galaxy. The other, at an observed wavelength of $592.55 \mathrm{~nm}$, is consistent with interstellar matter in NGC 4038 and implies a recession velocity of $1705 \pm$ $144 \mathrm{~km} \mathrm{~s}^{-1}$. This velocity agrees with that of the NASA/IPAC Extragalactic Database, $1642 \pm 12 \mathrm{~km} \mathrm{~s}^{-1}$ (Lauberts \& Valentijn 1989).

\subsection{Polarization}

The observed Stokes parameters and polarization are shown in Figure 4. Going from top to bottom, the panels show the observed flux spectrum, the observed polarization, the observed $Q$ and $U$ Stokes parameters, and the observed polarization angle.

Establishing the intrinsic polarization of the SN requires the subtraction of additional polarization sources such as the polarization caused by interstellar matter in our galaxy and the host (this is the so-called interstellar polarization (ISP)). We estimate a value for the ISP based on two assumptions. One is that the emission part of the strong P-Cygni profiles in the spectrum is intrinsically unpolarized. This should be so 


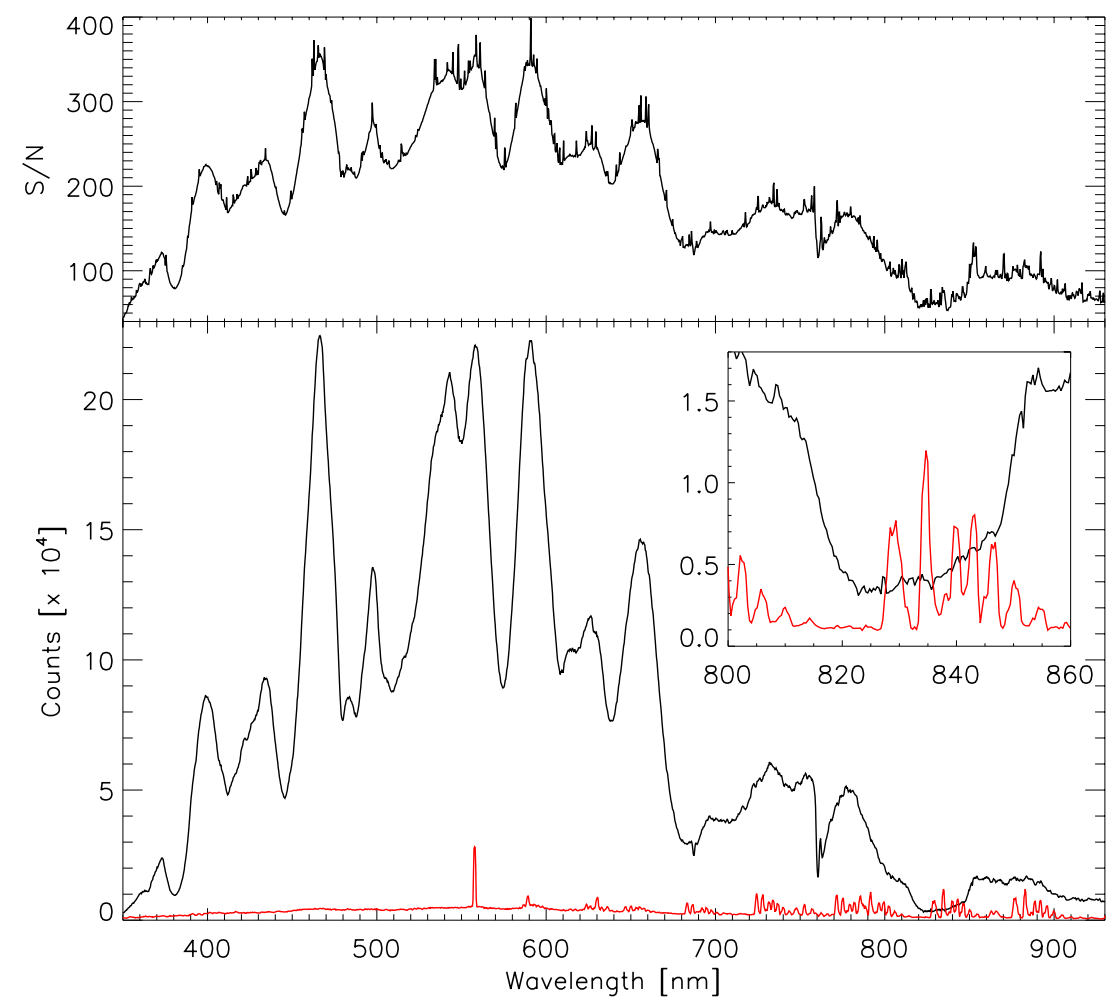

Figure 1. Intensity spectrum of SN 2007sr and the sky in counts (lower panel) and the S/N ratio (upper panel). In the region of the Ca II IR triplet (see the inset in the lower panel), the $\mathrm{S} / \mathrm{N}$ decreases significantly and the sky emission lines contaminate the red portion of the blueshifted absorption profile.

(A color version of this figure is available in the online journal.)

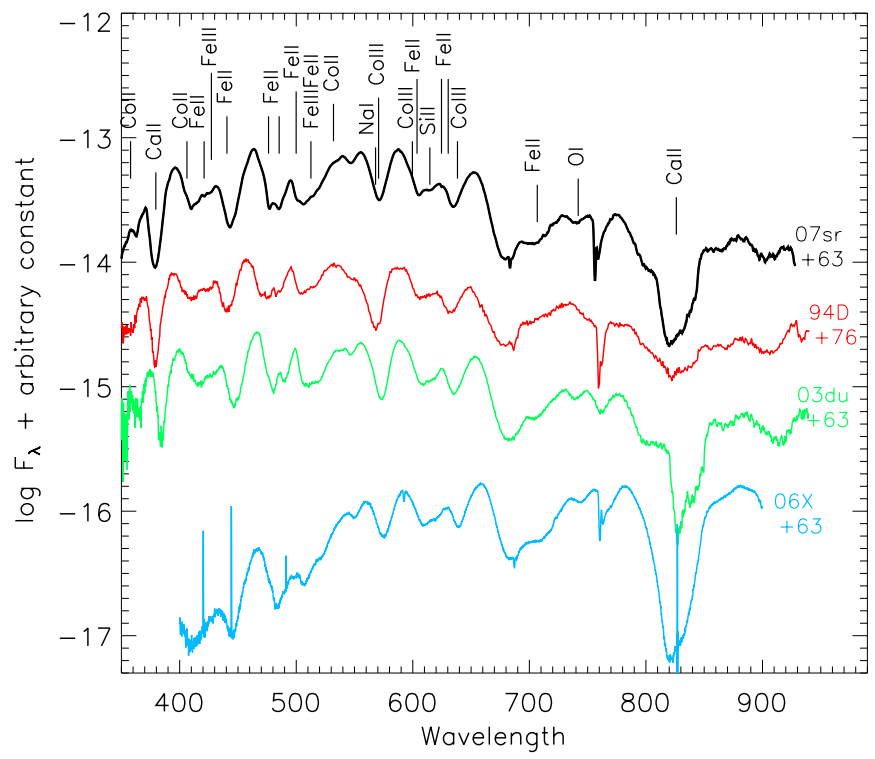

Figure 2. Comparison of the flux-calibrated spectrum of SN $2007 \mathrm{sr}$ with those of SNe 1994D, 2003du, and 2006X at similar phases. The data were obtained from the Online Supernova Spectrum Archive (SUSPECT) contributed by Gómez \& López (1998), Stanishev et al. (2007), and Yamanaka et al. (2009), respectively. All of these SNe exhibited high-velocity features $\left(\geqslant 20,000 \mathrm{~km} \mathrm{~s}^{-1}\right)$ at their early phases, which encourages us to infer the same behavior for SN 2007sr (see Section 4).

(A color version of this figure is available in the online journal.)

because they originate in the resonant scattering of photons coming from the photosphere, which are emitted with a random orientation of the plane of polarization. This spectrum has two good lines for estimating the ISP in this way: the blend of Na I D and Co III at a rest-frame wavelength $\sim 5700 \AA$ and the

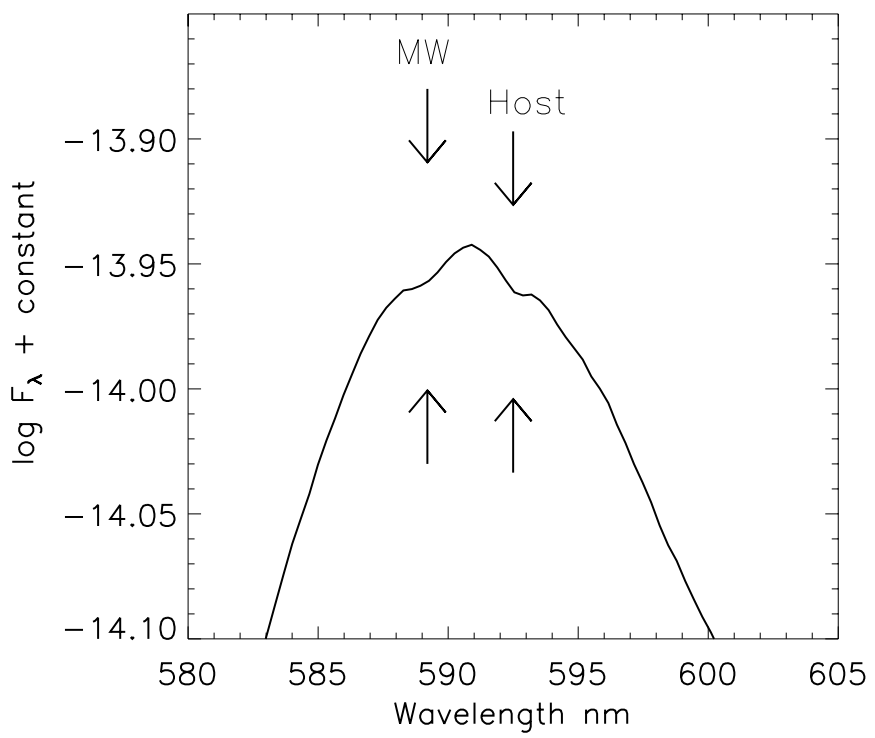

Figure 3. Na I D lines in the Milky Way and host galaxy at $589.19 \mathrm{~nm}$ and $592.55 \mathrm{~nm}$, respectively. The latter implies a recession velocity of $1705 \pm$ $144 \mathrm{~km} \mathrm{~s}^{-1}$, which agrees with the host velocity measured by Lauberts \& Valentijn (1989).

Ca II H\&K. The other assumption is that the blue side of the spectrum, the region between $\sim 400$ and $\sim 650 \mathrm{~nm}$ includes the blends of P-Cygni profiles of many different lines. Since the blending of P-Cygni lines should have a net depolarizing effect due to line blanketing opacity (Maund et al. 2010b), it is reasonable to assume zero intrinsic polarization in that part of the spectrum. Both assumptions consistently indicate an ISP with Stokes parameters $Q_{\mathrm{ISP}} \simeq 0$ and $U_{\mathrm{ISP}} \simeq 0.1\left(P_{\mathrm{ISP}}\right.$ $=0.1$. Finally, we assume a single Serkowski profile with 


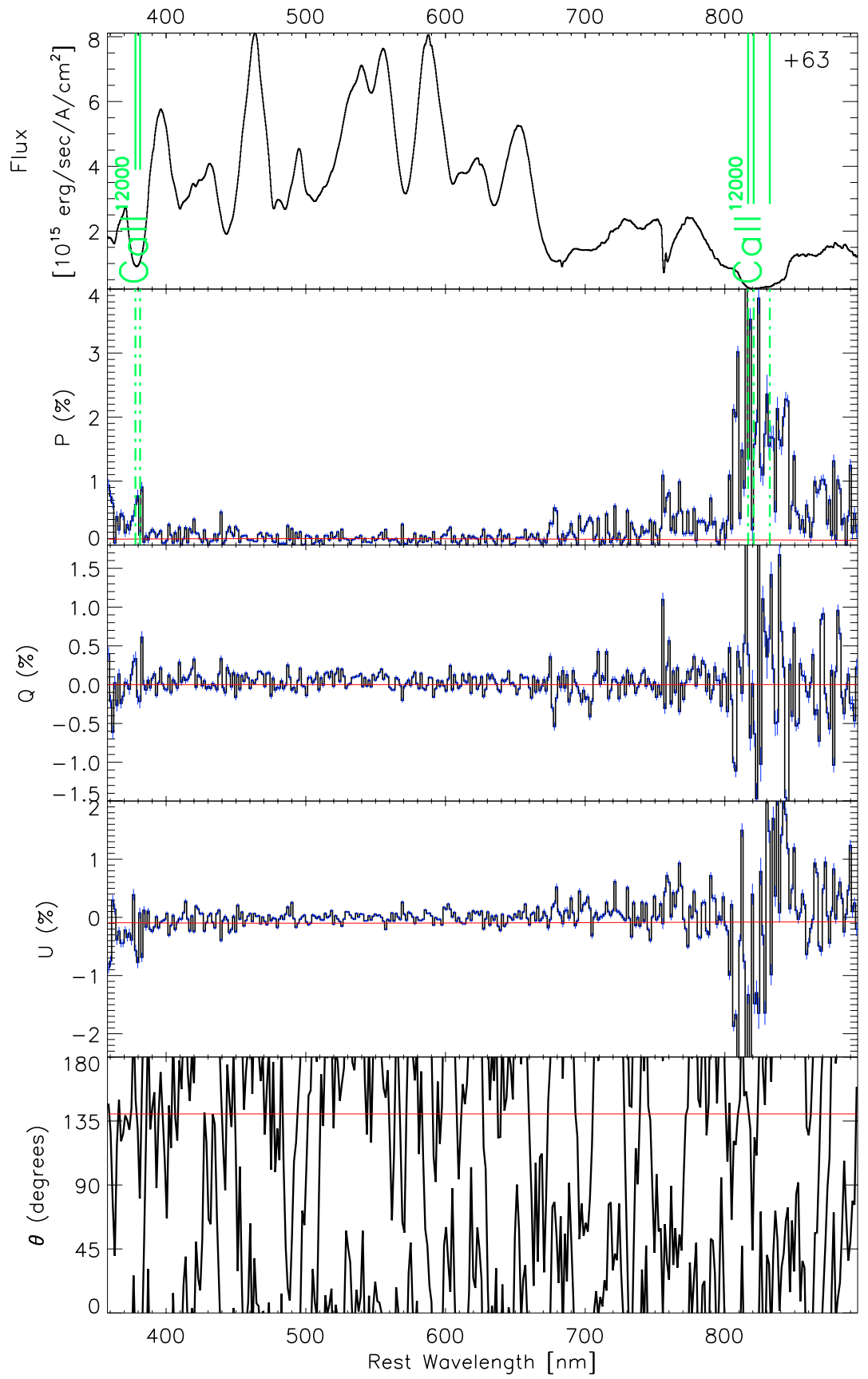

Figure 4. Observed flux, polarization, $Q$ and $U$ parameters vs. rest-frame wavelength. The Stokes parameters were computed using a bin size of $15 \AA$ in the observer frame. The horizontal red line shows a Serkowski law computed with $Q_{\mathrm{ISP}}=0$ and $U_{\mathrm{ISP}}=0.1$. Strong polarization is observed in the region of the Ca II IR triplet, and a less prominent feature appears in the region of Ca II H\&K. Peaks around $6800 \AA \mathrm{nm}$ and $7500 \AA$ are due to telluric absorption lines.

(A color version of this figure is available in the online journal.)

$\lambda_{\max }=5500 \AA$ as the wavelength dependence of the ISP (Serkowski et al. 1975). Panel 2 of Figure 4 shows the ISP (nearly horizontal red line), which confirms that the intrinsic polarization of the continuum is indeed consistent with zero.

We note that the previous ISP estimate is consistent with the observational relation between the color excess $E(B-V)$ and $P_{\text {ISP }}$ established by Serkowski et al. (1975), which takes the form of the upper limit $P_{\text {ISP, } \max } / E(B-V) \lesssim 9.0$. Both the color excess in the SN host measured by Schweizer et al. (2008) and the one in the Galaxy could justify an even larger ISP. We note as well that the equivalent width of the narrow $\mathrm{Na}$ I D lines in our spectrum and the color excess of Schweizer et al. give conditions for SN 2007sr for which both correlations in Turatto et al. (2003) are appropriate (see their Figure 3). Turatto's relations, which can be used to calculate two extreme values of $E(B-V)$, give the color excess $E(B-V)$ expected for a given EW of the Na I D lines. The two linear correlations they find are probably the result of different dust-to-gas ratios in the host galaxies.

In contrast to the null continuum polarization, there is line polarization in the Ca II features. With the spectrum binned to $15 \AA$, the Ca II H\&K and the infrared triplet display polarized components with maximum values of $\sim 1 \%$ and $\sim 4 \%$, respectively.

The polarization level is reduced by a factor of two, approximately, for a bin size of $30 \AA ̊$; see also Figures 5 and 6. In 

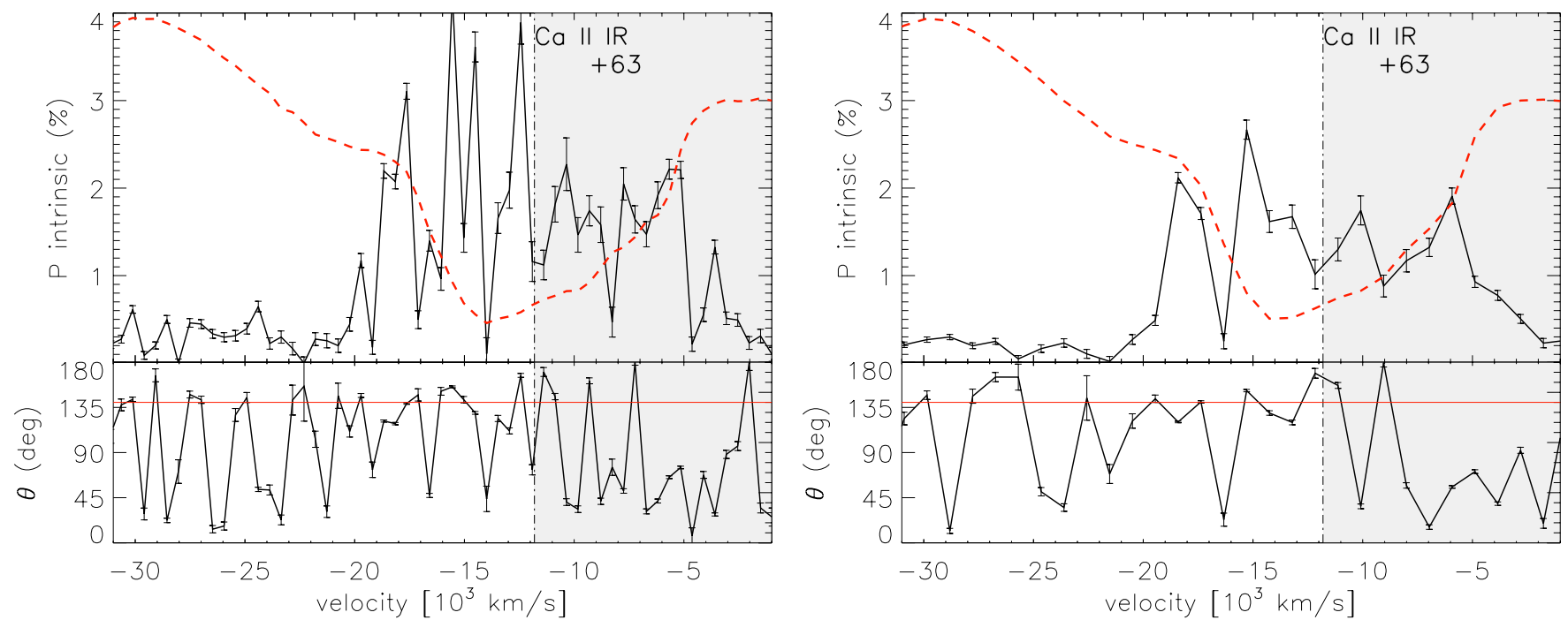

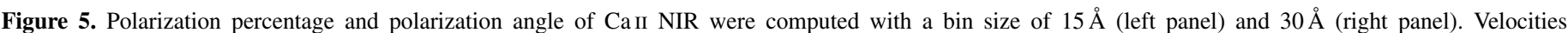

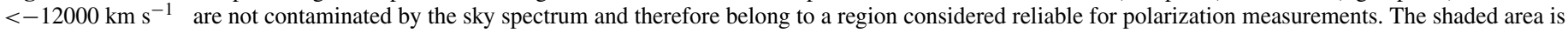
the range of wavelength highly contaminated by sky emission lines (see Figure 1).

(A color version of this figure is available in the online journal.)
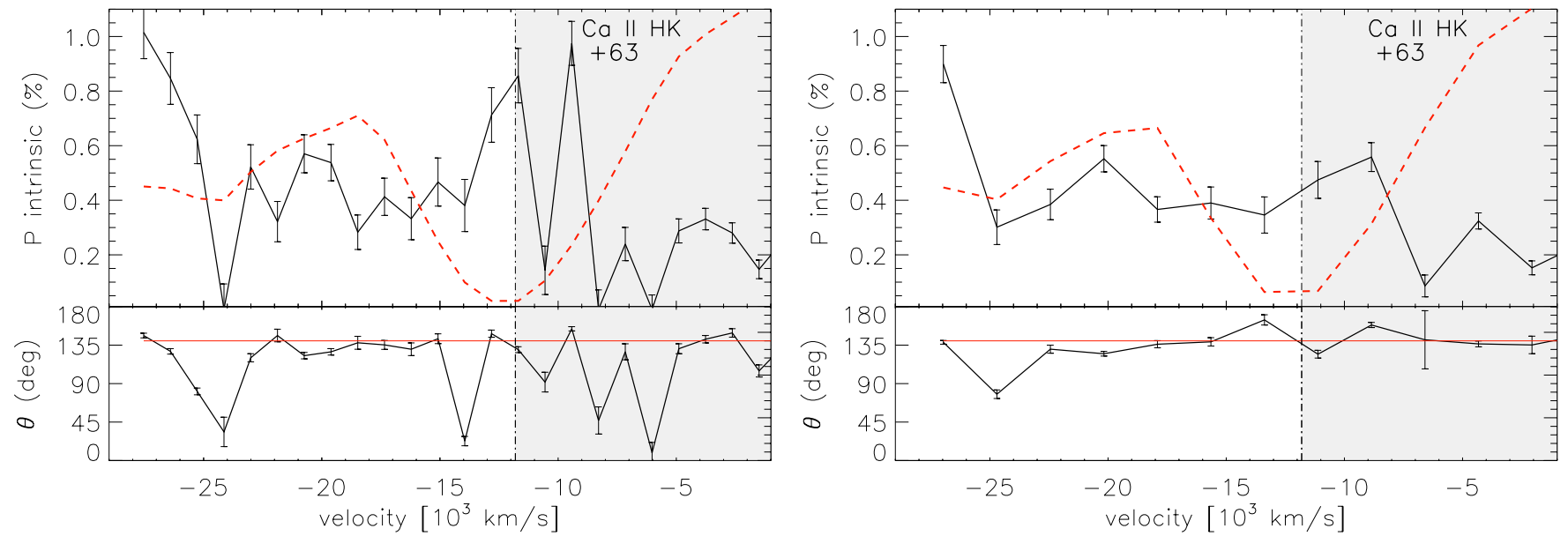

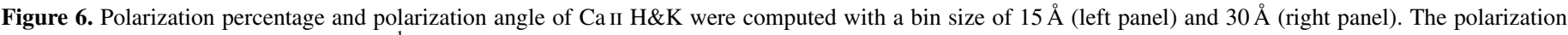

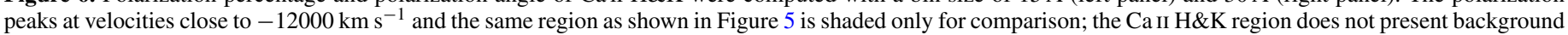
contamination.

(A color version of this figure is available in the online journal.)

any case, the polarization angle is close to $140^{\circ}$. As pointed out in Section 3.1, the Ca II lines extend between 5000 and $20000 \mathrm{~km} \mathrm{~s}^{-1}$. In the case of the Ca II IR triplet, the low S/N means a noisy signal. In the red part of the absorption profile, which corresponds to low expansion velocities, this could even result in some residual contamination from the, also highly polarized, sky background (see below). The high-velocity portion of the blueshifted absorption minimum, on the other hand, is cleaner. Finally, the Ca II H\&K lines do not have significant background contamination.

To understand the effects of the sky in the estimated polarization, we study the intrinsic polarization versus the intrinsic polarization angle at different wavelengths, as shown in Figure 7 for SN 2007sr and in Figure 8 for the sky. In Figure 7 we identify two well-defined angles with significant polarization, the first around $140^{\circ}$ and the second around $45^{\circ}$. The region of $140 \pm$ $30^{\circ}$, spanning most of the features of interest, has been colored gray. The red wavelength region, $800-890 \mathrm{~nm}$, which includes the Ca II IR triplet (red dots) displays components with signif- icant polarization at both angles. The blue wavelength region of the spectrum displays components with significant polarization only around $140^{\circ}$ (bluer dots with $\sim 1 \%$ polarization). As Figure 8 shows, the background sky that we subtracted from the spectra to get the intensity with each position of the half wave plate is also significantly polarized, with an angle close to $45^{\circ}$. In some parts of the red portion of the Ca II IR triplet the sky is brighter than the SN (see the inset in Figure 1); it is not surprising that there the observed polarization is very noisy and tends to recover the value of the sky. This is why we prefer not to give any weight to the polarization observed in the redder part of the Ca II IR triplet (red points at lower angles in Figure 7, outside of the gray region). We suspect that this conspicuous polarization feature is just residual contamination of the brighter subtracted sky, which happens to be polarized at about the same angle.

Recent work by Tanaka et al. (2012) showed that, for a sample of stripped-envelope $\mathrm{SNe}$, stronger lines exhibited higher polarization. They propose a simple radiative transfer model to relate the measured polarization to more physically 


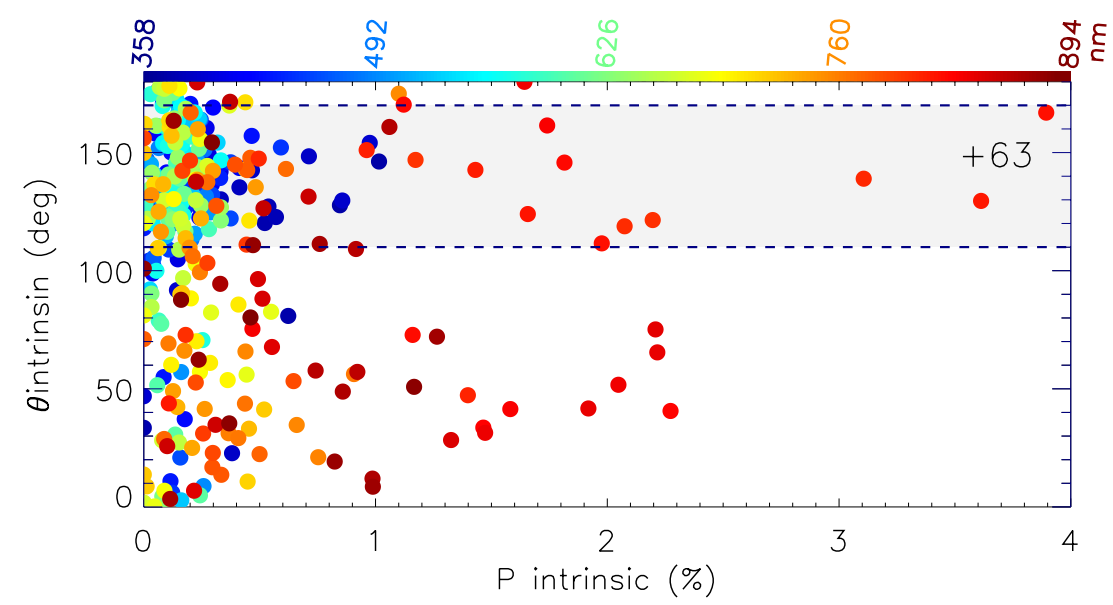

Figure 7. Polarization vs. polarization angle of SN2007sr (bin $=15 \AA$ ). Strong polarization is distributed around two angles, $140^{\circ}$ and $45^{\circ}$. The first one colored in gray includes Ca II, $\mathrm{H} \& \mathrm{~K}$ (blue dots), and the IR triplet (red dots). The $45^{\circ}$ angle corresponds to sky polarization, which is strong and contaminates part of the Ca II IR triplet profile (see Figure 8).

(A color version of this figure is available in the online journal.)

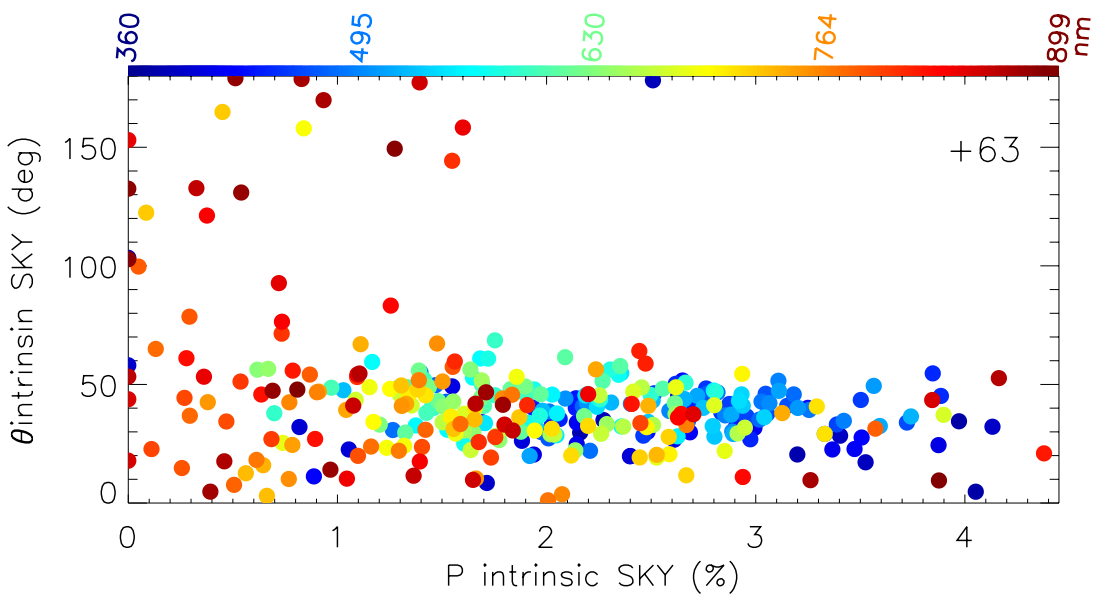

Figure 8. Polarization vs. polarization angle of the spectrum of the sky of SN $2007 \mathrm{sr}$ (bin $=15 \AA$ ). Sky polarization is probably dominated by moonlight polarization, at the observation date on which the moon was at an angular distance of $140^{\circ}$ away from the $\mathrm{SN}$, at first quarter phase. The sky polarization is distributed along the $45^{\circ}$ angle; in Figure 7 this corresponds to the lower angle zone, below the gray region.

(A color version of this figure is available in the online journal.)

meaningful parameters: an enhancement of absorption characterized by a factor $f$ and the fraction $\Delta S$ of the photospheric disk area $S$, covered by this enhancement. A combination of these two parameters is incorporated into a "corrected" line polarization, $P_{\text {corr }}$, which encodes the asymmetry that originates the observed line polarization. The latter, in addition, strongly depends on the strength of the absorption feature in the intensity spectrum, measured by the fractional depth with respect to the local continuum. This is interesting since it makes it possible to understand a diversity of polarization signals by resorting to a single anisotropy and a variety of absorption line strengths.

Although the model of Tanaka et al. (2012) was imagined and tested for core-collapse $\mathrm{SNe}$, it is general enough to be readily applied to Type Ia SNe. We have done so and the results of the exercise are shown in Figure 9. Measuring the fractional depth requires estimating the continuum at the position of the absorption feature. For the complex late spectrum of a Type Ia $\mathrm{SN}$, this is difficult to do without a detailed radiative transfer model. This is especially true in the case of the Ca II IR triplet, where the uncertainty in identifying the continuum leads to a fairly large uncertainty in the fractional depth, even though it is possible to see in Figure 9 that the polarization signals observed in both Ca II lines are mutually consistent. Given the large difference in fractional depths, the $\sim 1 \%$ polarization observed for $\mathrm{Ca}$ II $\mathrm{H} \& \mathrm{~K}$ translates into a $2 \%-4 \%$ polarization in the CaII IR triplet, for a modest value of the intrinsic polarization. This reinforces the view that $\mathrm{Ca}$ II is intrinsically polarized and that a single anisotropy is responsible for both signals.

The intrinsic $Q-U$ diagram of the entire spectrum is shown in Figure 10. The scatter of points around $(Q, U)=(0,0)$ reveals an almost null global polarization. There is a peculiar quasi-spiral loop in the region of the Ca IR triplet, which, as mentioned earlier, we understand to be due to the mixture of intrinsic Ca II IR triplet polarization and sky polarization. As in Figure 7 we have colored the region gray where the intrinsic line polarization of the $\mathrm{SN}$ is located. Figure 11 gives the $Q U$ diagram of the region of $\mathrm{Ca}$ II in velocity space, the IR triplet region is represented by filled circles, and the H\&K region is represented by stars. Cyan and blue points correspond to velocities higher than $13000 \mathrm{~km} \mathrm{~s}^{-1}$, uncontaminated by the sky in the case of IR triplet. It is precisely at those velocities where the strong polarization signals are measured and seem to be concentrated in the gray area (between $110^{\circ}$ and $170^{\circ}$ ) for both Ca II lines. 


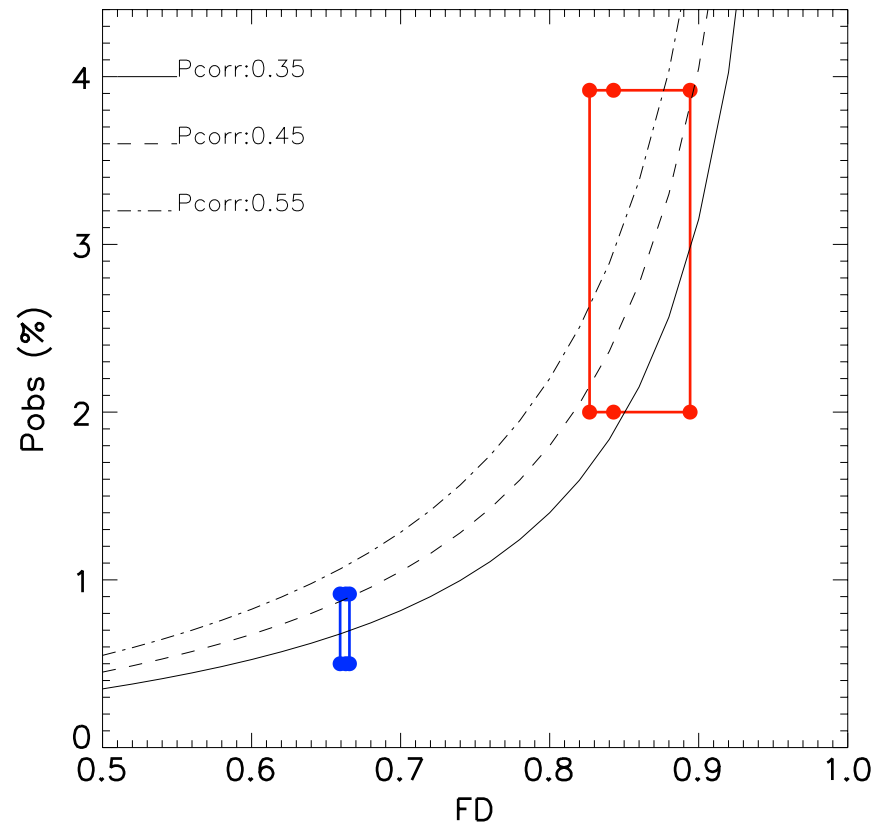

Figure 9. Observed line polarization of the Ca II features as a function of the fractional depth (FD) of the absorption line in the intensity spectrum, based on the work of Tanaka et al. (2012). Three estimates of FD for the Ca II H\&K line (in blue) and the Ca II IR triplet (in red) are given, corresponding to an upper, a lower, and an average estimate of the local continuum. The varying line polarization at each line is illustrated by the vertical lines, between $2 \%$ and $4 \%$ for the infrared triplet and between $0.5 \%$ and $1 \%$ for the $\mathrm{H} \& \mathrm{~K}$ lines. The plotted lines correspond to Equation (8) in Tanaka et al. (2012) for different values of the parameter that they call corrected polarization $\left(P_{\text {corr }}\right)$. The model is very simple, and the uncertainties are large, but it is nevertheless reassuring to find that the very different values of the observed polarization in the two calcium lines are consistent with a single value of $P_{\text {corr }}$, around $0.35-0.45$.

(A color version of this figure is available in the online journal.)

\section{DISCUSSION AND CONCLUSION}

Umbriaco et al. (2007) found high velocities in a spectrum of SN 2007sr taken closer to maximum light and suggested that the SN matched the spectra of HVG SNe (Benetti et al. 2005); further confirmed by Maeda et al. (2010a) and S. Benetti (2011, private communication). Consistent with this, our spectrum of SN 2007sr at 63 days after maximum light reveals high expansion velocities. The most prominent absorption lines that exhibit fast velocities are those of Ca II, which span the range 5-18 $\times 10^{3} \mathrm{~km} \mathrm{~s}^{-1}$. High velocities at late phases are consistent with the existence of high-velocity components prior to maximum light, as was the case for SN 2004dt and SN 2006X (Wang et al. 2006; Patat et al. 2009). All the SNe used for comparison spectra in Figure 2 (SN 1994D, 2003du, and 2006X) showed high-velocity components in their early phases, which also makes it tempting to deduce that SN2007sr must have had high velocity features (HVFs) in its early phases.

The very low polarization of the continuum adds one more case to consolidate the standard view that normal Type Ia SNe have only small, if any, departures from global spherical symmetry, in particular toward later epochs when we see deeper layers (Filippenko \& Leonard 2004; Leonard et al. 2005; Wang $\&$ Wheeler 2008). The line polarization, however, shows a different picture. The usual expectation is that line polarization, if present at early epochs, will decrease and disappear at later phases. The best-studied line is Si II 6355. In most SNe observed around maximum light, and few days or weeks after maximum, the polarization of the line peaks around maximum and then disappears in a timescale of weeks (Wang et al. 2007). The same behavior has been reported in the polarization of $\mathrm{Ca}$ II lines. SN 2001el showed a high-velocity Ca II IR triplet with a maximum line polarization of $\sim 0.8 \%$ at early times. This polarization decreased after maximum and was very small at a phase of 38 days (Wang et al. 2003).
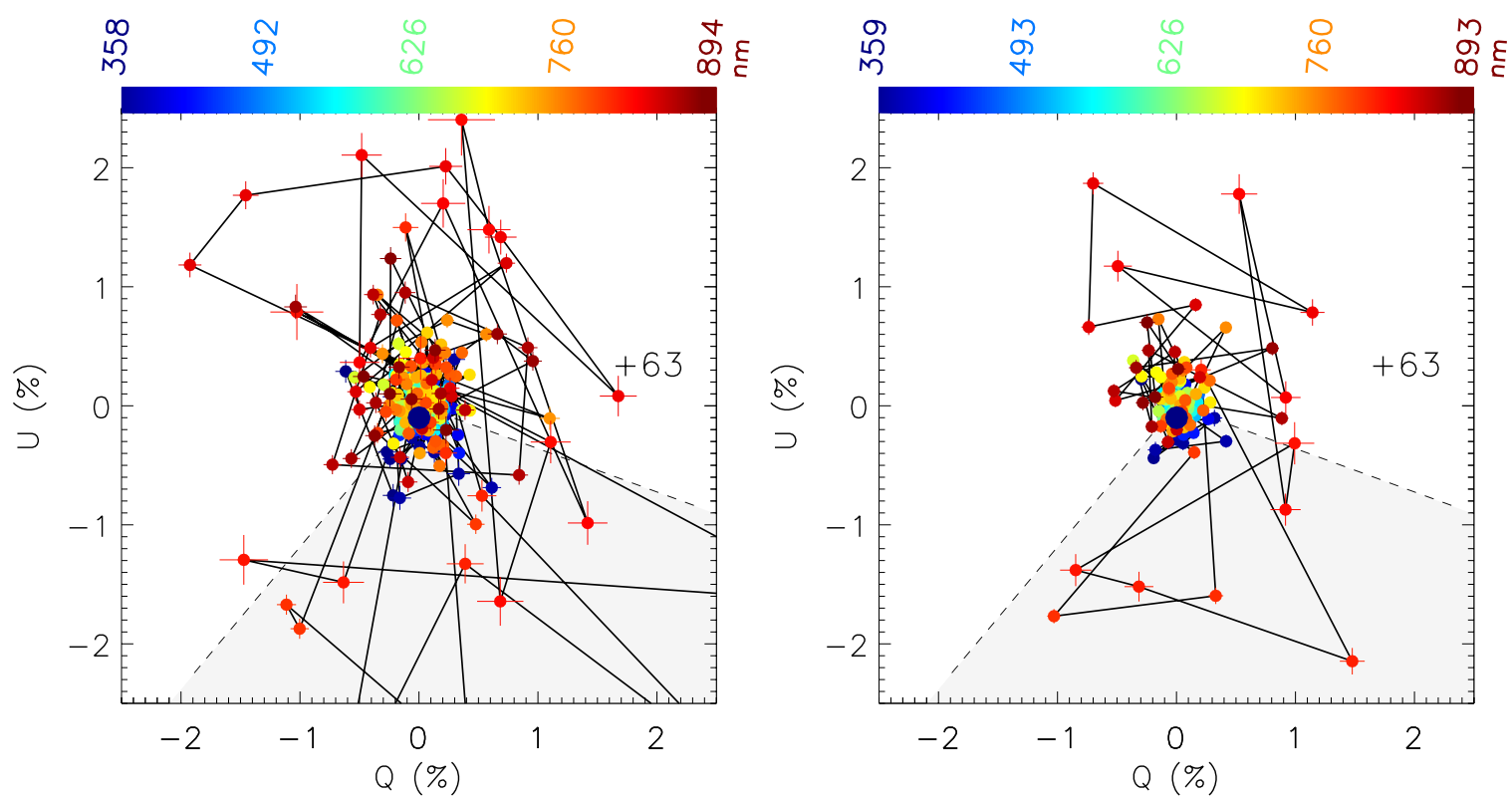

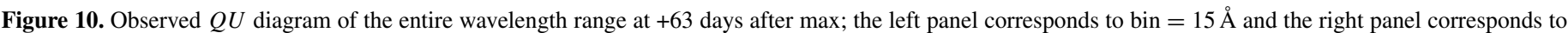

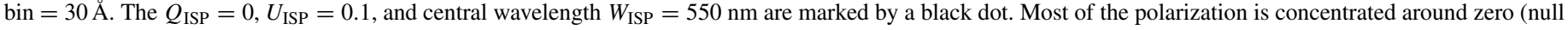

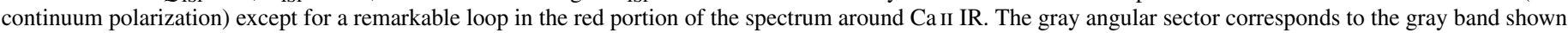
in Figure 7.

(A color version of this figure is available in the online journal.) 

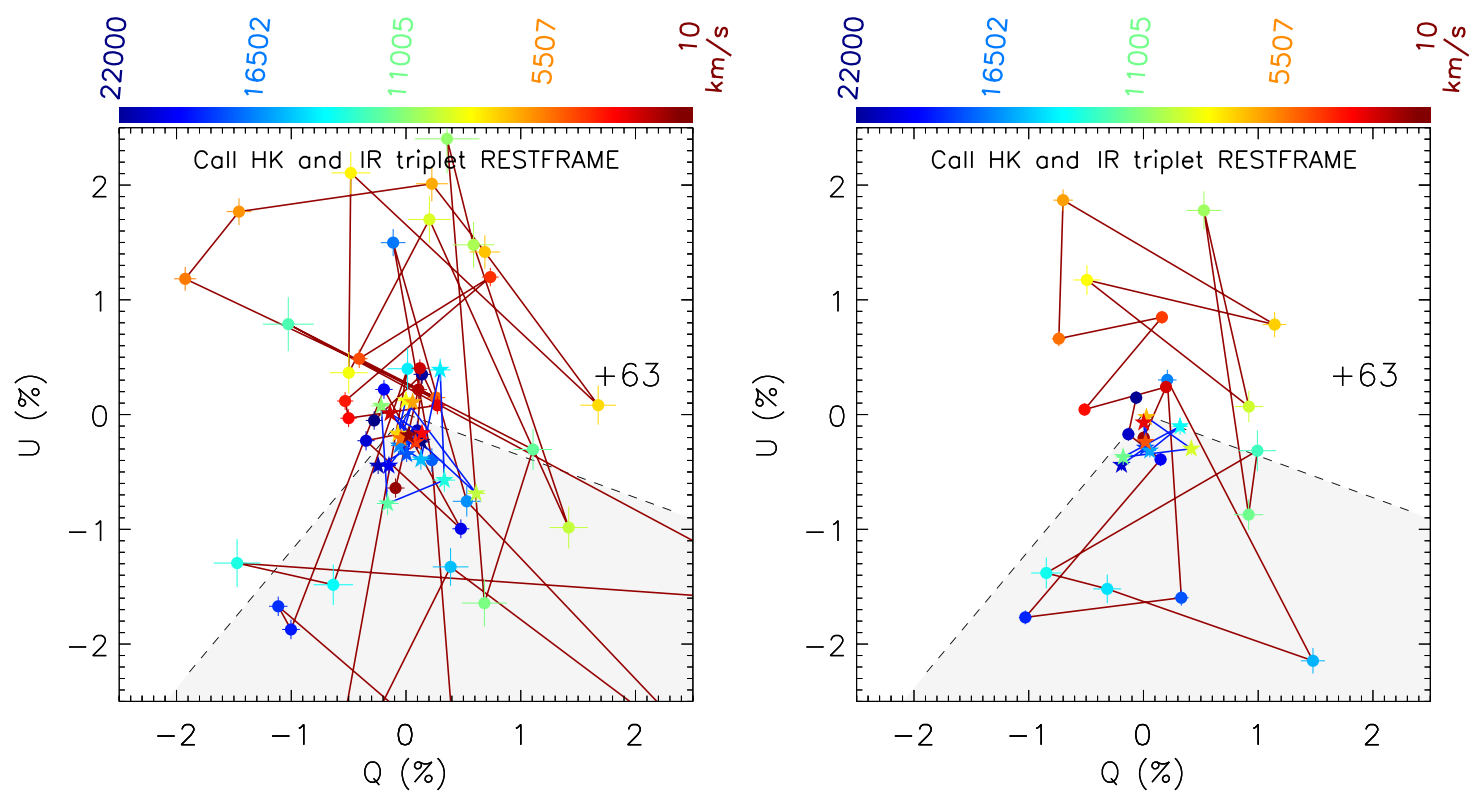

Figure 11. Observed $Q U$ diagram of the Ca II IR region, showing a loop of high velocities (the left panel corresponds to bin $=15 \AA$ and the right panel to bin $=30 \AA$ ). Blue and cyan dots correspond to the velocity range $20000-13000 \mathrm{~km} \mathrm{~s}^{-1}$ where the polarization measurements are uncontaminated by the sky, and they are also the highest polarization values. The gray angular sector corresponds to the gray band shown in Figure 7.

(A color version of this figure is available in the online journal.)

It appears, however, that the Ca II lines do not always follow this tendency. SN 2007sr exhibits strong polarization in Ca II two months after maximum light, and it occurs in a rapidly expanding feature. Patat et al. (2009) found high late-time polarization of the Ca II IR triplet in SN 2006X, another rapidly expanding event observed from early phases until 39 days after maximum light. They describe a re-polarization of the line and, based on the abundance distribution of a delayed detonation model of Höflich et al. (2002), interpret it to be a partial cover-up of the photosphere in the line wings, where the Sobolev optical depth is smaller. They also point out that the lack of significant line polarization at the inner Ca boundary $\left(v \sim 10000 \mathrm{~km} \mathrm{~s}^{-1}\right.$ for the same model) is a probable sign of mixing at that inner edge. Due to the contamination by the sky background, we cannot trace the polarization of the Ca II IR triplet to these low velocities. The Ca II H\&K lines, however, which give a more certain estimate of the intrinsic polarization, allow us to see the same effect in SN 2007sr. The line polarization decreases sharply for velocities between 9000 and $8000 \mathrm{~km} \mathrm{~s}^{-1}$, which will be consistent with mixing up to a lower velocity.

Having just one spectrum of SN 2007sr, we do not know if the high polarization of $\mathrm{Ca}$ II is a re-polarization at late times or if Ca II was always polarized from maximum light onward. It is important to note that SN 2007sr adds to the case that some Type Ia $\mathrm{SNe}$ that are considered to be normal do show high line polarization at late phases. We do not know why some do and some others do not show this late polarization. From the data at hand, however, it is tempting to connect this late-time line polarization with the presence of HV Ca II features. If this were the case, the late-time polarization could be due to the interaction of the ejecta with circumstellar matter as suggested by Gerardy et al. (2004).

Few SNe have been observed with spectropolarimetry at phases as late as SN 2006X and 2007sr. We have already mentioned SN 2001el; another case is SN 2005ke, a subluminous event, which did not show $\mathrm{Ca}$ II polarization to a statistically significant level either before maximum or at $\sim 76$ days after maximum (Patat et al. 2012). It is important to increase the database. A larger, good quality data set will allow us to connect this observable with others, such as the presence of HVFs, and, eventually, will help us to disentangle the effects of the explosion physics from those of the aspect angle or those of the interaction between the ejecta and circumstellar matter.

P.Z., J.Q., \& A.C. acknowledge support by Iniciativa Cientifica Milenio (MINECON, Chile) through the Millennium Center for Supernova Science (P10-064-F). P.Z. also acknowledges CONICYT, Chile (Beca de Doctorado). The research of J.R.M. is supported through a Royal Society University Research Fellowship. A.C. acknowledges support through grants Basal CATA PFB 06/09 and FONDAP No. 15010003 from CONICYT, Chile. P.A.H. was supported by the NSF grants 0703902 and 0708855.

\section{REFERENCES}

Benetti, S., Cappellaro, E., Mazzali, P. A., et al. 2005, ApJ, 623, 1011 Branch, D., Baron, E., Hall, N., Melakayil, M., \& Parrent, J. 2005, PASP, 117,545

Branch, D., Fisher, A., \& Nugent, P. 1993, AJ, 106, 2383

Branch, D., Jeffery, D. J., Parrent, J., et al. 2008, PASP, 120, 135

Burns, C. R., Stritzinger, M., Phillips, M. M., et al. 2011, AJ, 141, 19

Drake, A. J., Djorgovski, S. G., Williams, R., et al. 2007, CBET, 1172, 1

Fesen, R. A., Höflich, P. A., Hamilton, A. J. S., et al. 2007, ApJ, 658, 396

Filippenko, A. V., \& Leonard, D. C. 2004, in Cosmic Explosions in Three Dimensions: Asymmetries in Supernovae and Gamma-Ray Bursts, ed. P. Höflich, P. Kumar, \& J. C. Wheeler (Cambridge Contemporary Astrophysics Series; Cambridge: Cambridge Univ. Press), 30

Folatelli, G., Phillips, M. M., Burns, C. R., et al. 2010, AJ, 139, 120

Gerardy, C. L., Höflich, P., Fesen, R. A., et al. 2004, BAAS, 36, 777

Gómez, G., \& López, R. 1998, AJ, 115, 1096

Hamuy, M., Phillips, M. M., Suntzeff, N. B., et al. 1996, AJ, 112, 2408

Hamuy, M., Trager, S. C., Pinto, P. A., et al. 2000, AJ, 120, 1479

Hatano, K., Branch, D., Fisher, A., Baron, E., \& Filippenko, A. V. 1999, ApJ, 525,881

Hillebrandt, W., Niemeyer, J. C., \& Reinecke, M. 2000, in AIP Conf. Ser. 522, Cosmic Explosions (Melville, NY: AIP), 53

Höflich, P. 1991, A\&A, 246, 481

Höflich, P., Gerardy, C. L., Fesen, R. A., \& Sakai, S. 2002, ApJ, 568, 791

Höflich, P., \& Khokhlov, A. 1996, ApJ, 457, 500 
Howell, D. A., Höflich, P., Wang, L., \& Wheeler, J. C. 2001, ApJ, 556, 302

Ivanov, V. D., Hamuy, M., \& Pinto, P. A. 2000, ApJ, 542, 588

Kasen, D., Röpke, F. K., \& Woosley, S. E. 2009, Natur, 460, 869

Kasen, D., Thomas, R. C., Röpke, F., \& Woosley, S. E. 2008, JPhCS, 125, 012007

Khokhlov, A. M. 1991, A\&A, 245, L25

Lauberts, A., \& Valentijn, E. A. 1989, Msngr, 56, 31

Leonard, D. C., Li, W., Filippenko, A. V., Foley, R. J., \& Chornock, R. 2005, ApJ, 632,450

Liu, W., Jeffery, D. J., Schultz, D. R., et al. 1997, ApJ, 489, L141

Madore, B. F., \& Steer, I. P. 2007, Master List of Galaxy Distances (Rev. 2.2), http://ned.ipac.caltech.edu/level5/NED1D/intro.html

Maeda, K., Benetti, S., Stritzinger, M., et al. 2010a, Natur, 466, 82

Maeda, K., Taubenberger, S., Sollerman, J., et al. 2010b, ApJ, 708, 1703

Maund, J. R., Höflich, P., Patat, F., et al. 2010a, ApJ, 725, L167

Maund, J. R., Wheeler, J. C., Wang, L., et al. 2010b, ApJ, 722, 1162

Patat, F., Baade, D., Höflich, P., et al. 2009, A\&A, 508, 229

Patat, F., Höflich, P., Baade, D., et al. 2012, A\&A, 545, A7

Patat, F., Möhler, S., O’Brien, K., et al. 2011, A\&A, 527, A91

Patat, F., \& Romaniello, M. 2006, PASP, 118, 146

Phillips, M. M. 1993, ApJ, 413, L105
Pojmanski, G., Prieto, J. L., Stanek, K. Z., \& Beacom, J. F. 2008, CBET, 1213,1

Prieto, J. L., Rest, A., \& Suntzeff, N. B. 2005, in ASP Conf. Ser. 339, Observing Dark Energy, ed. S. C. Wolff \& T. R. Lauer (San Francisco, CA: ASP), 69

Saviane, I., Momany, Y., da Costa, G. S., Rich, R. M., \& Hibbard, J. E. 2008, ApJ, 678,179

Schlegel, D. J., Finkbeiner, D. P., \& Davis, M. 1998, ApJ, 500, 525

Schweizer, F., Burns, C. R., Madore, B. F., et al. 2008, AJ, 136, 1482

Serkowski, K., Mathewson, D. S., \& Ford, V. L. 1975, ApJ, 196, 261

Stanishev, V., Goobar, A., Benetti, S., et al. 2007, A\&A, 469, 645

Tanaka, M., Kawabata, K. S., Hattori, T., et al. 2012, ApJ, 754, 63

Tonry, J. L., Dressler, A., Blakeslee, J. P., et al. 2001, ApJ, 546, 681

Turatto, M., Benetti, S., \& Cappellaro, E. 2003, in ESO Astrophys. Symp., From Twilight to Highlight: The Physics of Supernovae, ed. W. Hillebrandt \& B. Leibundgut (Berlin: Springer), 200

Umbriaco, G., Pietrogrande, T., di Mille, F., et al. 2007, CBET, 1174, 1

Wang, L., Baade, D., Höflich, P., et al. 2003, ApJ, 591, 1110

Wang, L., Baade, D., Höflich, P., et al. 2006, ApJ, 653, 490

Wang, L., Baade, D., \& Patat, F. 2007, Sci, 315, 212

Wang, L., \& Wheeler, J. C. 2008, ARA\&A, 46, 433

Yamanaka, M., Naito, H., Kinugasa, K., et al. 2009, PASJ, 61, 713 